ESAIM: PROCEEDINGS, Juillet 2013, Vol. 40, p. 1-15

C. Bourdarias, S. Gerbi, Editors

\title{
SOLUTIONS FOR A HYPERBOLIC MODEL OF MULTI-PHASE FLOW
}

\author{
Debora Amadori ${ }^{1}$ AND ANDrea CORLI ${ }^{2}$
}

\begin{abstract}
We discuss a model for the flow of an inviscid fluid admitting liquid and vapor phases, as well as a mixture of them. The flow is modeled in one spatial dimension; the state variables are the specific volume, the velocity and the mass density fraction $\lambda$ of vapor in the fluid. The equation governing the time evolution of $\lambda$ contains a source term, which enables metastable states and drives the fluid towards stable pure phases.

We first discuss, for the homogeneous system, the BV stability of Riemann solutions generated by large initial data and check the validity of several sufficient conditions that are known in the literature.

Then, we review some recent results about the existence of solutions, which are globally defined in time, for $\lambda$ close either to 0 or to 1 (corresponding to almost pure phases). These solutions possibly contain large shocks. Finally, in the relaxation limit, solutions are proved to satisfy a reduced system and the related entropy condition.
\end{abstract}

Résumé. On discute un modèle pour l'écoulement d'un fluide non visqueux admettant phases liquides et de vapeur, ainsi qu'un mélange d'entre eux. L'écoulement est modélisé dans une dimension spatiale; les variables d'état sont le volume spécifique, la vitesse et la fraction de densité de masse $\lambda$ de la vapeur dans le liquide. L'équation régissant l'évolution temporelle de $\lambda$ contient un terme de source, ce qui permet des états métastables et conduit le fluide vers de phases stables pures.

Nous discutons d'abord, pour le système homogène, la stabilité BV des solutions de Riemann générés par des grandes données initiales et vérifions la validité de plusieurs conditions suffisantes qui sont connues dans la littérature.

Ensuite, nous passons en revue quelques résultats récents sur l'existence de solutions, qui sont definies pour tous les temps, pour $\lambda$ soit près de 0 ou de 1 (correspondant à des phases presque pures). Ces solutions sont susceptibles de contenir des grands chocs. Enfin, dans la limite de la relaxation, les solutions sont prouvèes satisfaire un système réduit et la condition d'entropie.

\section{INTRODUCTION}

We consider here a simple hyperbolic model for the flow of a fluid capable of showing a liquid and a gaseous phase. A reaction term is also taken into consideration, which allows for metastable states. In Lagrangian variables the model is represented by the following system of balance laws:

$$
\begin{cases}v_{t}-u_{x} & =0 \\ u_{t}+p(v, \lambda)_{x} & =0 \\ \lambda_{t} & =\frac{1}{\tau}\left(p-p_{e}\right) \lambda(\lambda-1) .\end{cases}
$$

${ }^{1}$ Department of Engineering, Computer Science and Mathematics, University of L'Aquila, Italy

2 Department of Mathematics and Computer Science, University of Ferrara, Italy

(C) EDP Sciences, SMAI 2013 
ESAIM: PROCEEDINGS

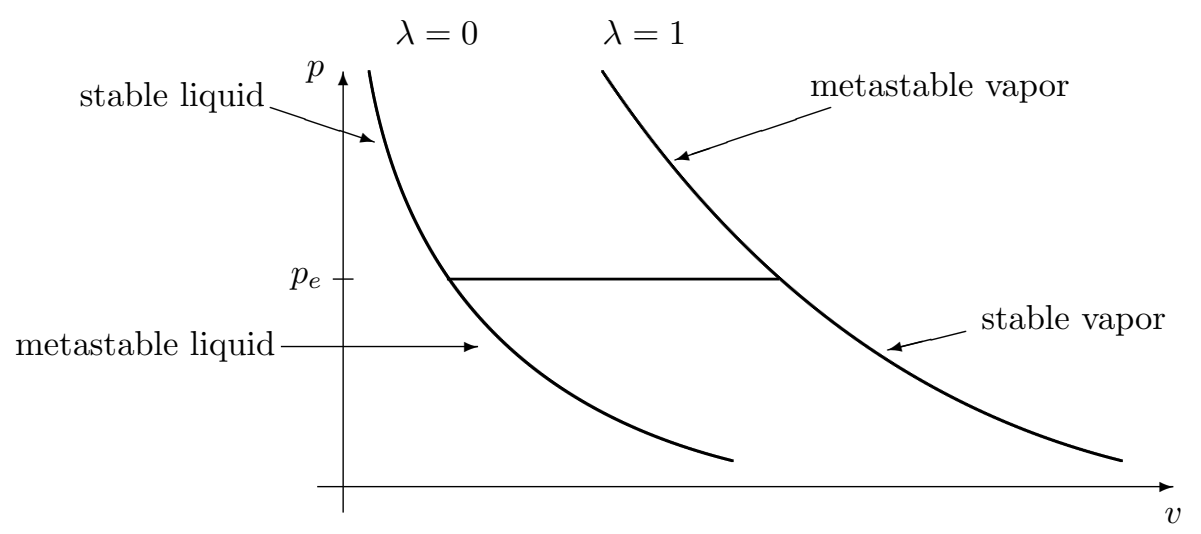

FiguRE 1. Pressure curves.

Here $v>0$ is the specific volume and $u$ the velocity. The state variable $\lambda$ is the mass density fraction of the vapor in the fluid; as a consequence, we have that $0 \leq \lambda \leq 1$. The value $\lambda=0$ then refers to the liquid phase while the value $\lambda=1$ to the vapor (gaseous) phase; intermediate values describe suitable mixtures of the previous pure phases. In the last equation, $p_{e}$ denotes a fixed equilibrium pressure and $\tau>0$ is a reaction time.

An important feature of the model consists in the fact that the pressure $p$ is allowed to depend not only on $v$ but also on $\lambda$. More precisely, we assume that

$$
p=p(v, \lambda)=A(\lambda) \pi(v), \quad A(\lambda)>0, \quad \pi(v)>0
$$

where $A, \pi$ are $C^{2}$ functions that satisfy

$$
A^{\prime}(\lambda) \neq 0, \quad \pi_{v}<0, \quad \pi_{v v}>0 .
$$

Under the above assumptions, the homogeneous part of (1) turns out to be strictly hyperbolic. The eigenvalues are $\pm \sqrt{-p_{v}}$ and 0 ; the two former are genuinely nonlinear and the latter is linearly degenerate.

A typical example for $\pi$ is the usual $\gamma$-law for the isentropic (isothermal) gasdynamics, i.e., $\pi(v)=1 / v^{\gamma}$ with $\gamma \geq 1$. For $A(\lambda)$ a possible choice is a linear interpolation between the two pure phases; namely, $A(\lambda)=$ $k_{0}+\lambda\left(k_{1}-k_{0}\right)$, with $0<k_{0}<k_{1}$. Of course, in the case that $A$ is constant the model reduces to the standard $p$-system in gasdynamics. A motivation for the pressure to depend on $\lambda$ can be given by considering a pressure law for real gases (the van der Waals pressure law, for instance) when the temperature is above the critical point. In such cases what is really observable are the two hyperbolic branches of the pressure associated to the liquid and to the vapor phase; in our model they are represented by $A(0) \pi(v)$ and $A(1) \pi(v)$, respectively. The pressure curves $A(\lambda) \pi(v)$, for $0<\lambda<1$, interpolate the curves of the pressure in the liquid and vapor phases in the case of mixtures.

The model (1) was first introduced in [21]; indeed the system in [21] is much more complete, including the fluid viscosity, the diffusion of the vapor in the fluid, nucleation terms and thermal effects. As a consequence, the analytic study of that model is extremely hard and some simplifications are demanded, as it is the case for (1). We notice that system (1) is isothermal, an assumption that can be done when studying phase changes in retrograde fluids, as a consequence of the their large heat capacities [44].

The reaction term on the right-hand side of the third equation in (1) allows for metastable states, because of the presence of the equilibrium pressure $p_{e}$ : namely, metastable states are the vapor states lying above the line $p=p_{e}$ or the liquid states lying below it. The line $p=p_{e}$, in the $(v, p)$ plane, then plays the role of the equal-area Maxwell line in the standard theory of phase transitions for fluids [43]. A model analogous to (1) but also including a damping term $-\alpha u$ in the second equation to model a flow through a porous medium, has 
been recently studied in [16]. Such flows occur, for instance, in the process of oil recovery from porous rocks or in the operation of a polymer electrolyte fuel cell, where a gaseous phase may develop as a consequence of high temperatures. About that model we also refer to [32], where however a different pressure law is considered. A close system is also studied in $[32,33]$; in those papers the third equation includes a transport term and becomes $\lambda_{t}+c_{1} \lambda_{x}=\beta(h(v)-\lambda) / \tau$, for $\beta$ constant and a suitable function $h$.

It is also interesting to compare (1) with a system proposed in [29] to model reacting flows. In that paper the term $p-p_{e}$, which leads to metastability, is missing and the third equation in (1) turns out be $\lambda_{t}=(\lambda(v)-\lambda) / \tau$, for a suitable positive and decreasing function $\lambda(v)$, while the pressure can be written under the form (2) and satisfies (3). Also the paper [22] deals with a third equation of the form $\lambda_{t}=g(\lambda)$, for a given function $g$, and shows the existence of solutions by compensated compactness; however, the pressure law is different from (2). A class of systems similar to (1) and modeling phase transition flows has been thoroughly studied in $[1,13,24,35]$, with particular emphasis to numerical simulations. Those models include also an equation for the conservation of energy and are written in Eulerian coordinates; the pressure is assigned according to the so-called stiffened gas pressure law $[1,13]$.

If the reaction term in the third equation of (1) is missing, we obtain the homogeneous system

$$
\begin{cases}v_{t}-u_{x} & =0 \\ u_{t}+p(v, \lambda)_{x} & =0 \\ \lambda_{t} & =0 .\end{cases}
$$

It is interesting to notice that the phase interfaces for system (4) are (stationary) contact discontinuities. This is in striking contrast with other models [14], where the phase interfaces are modeled by undercompressive shock waves. In such models the Riemann problem is underdetermined; the addition of a further external condition, the so called kinetic condition [28], which prescribes the speed of the interface, is required to single out solutions. For system (4), the fact that the phase interfaces are stationary can be understood as a kinetic condition. We refer to [3] for several comparison of the model (4) with other models for diphasic flows.

Here, our first aim is the study of the Cauchy problem for the system (1), in the case that the relaxation time $\tau>0$ is fixed, for the following initial data:

$$
(v, u, \lambda)(0, x)=\left(v_{o}(x), u_{o}(x), \lambda_{o}(x)\right) .
$$

We focus on the existence of weak solutions that exist for all times. Motivated by [37] we assume that

$$
\pi(v)=\frac{1}{v} .
$$

In the spirit of the fractional step method ( $[20])$, the analysis begins with the study of the homogeneous system (4); we shall refer to $[2,3]$. In particular, in [3] it is proved that solutions defined globally in time do exist for initial data having suitably bounded (but not necessarily small) total variation; as a consequence, large shocks can possibly show up in the solution. More precisely, the conditions in [3] require that $A_{o} \doteq \mathrm{TV}\left(\log a_{o}\right)<1 / 2$ and that

$$
\mathrm{TV}\left(\log p_{o}\right)+\frac{1}{\inf a_{o}} \mathrm{TV}\left(u_{o}\right)<H\left(A_{o}\right) .
$$

Here we denoted $a(\lambda)=\sqrt{A(\lambda)}, a_{o}=a\left(\lambda_{o}\right)$ and $p_{o}=p\left(v_{o}, \lambda_{o}\right)$. The function $H:(0,1 / 2] \rightarrow[0,+\infty)$, which has an explicit expression, satisfies $H(1 / 2)=0$ and $H(A) \rightarrow+\infty$ when $A \rightarrow 0+$. This roughly means that if $\operatorname{TV}\left(a\left(\lambda_{o}\right)\right)$ is small then both $\mathrm{TV}\left(\log p_{o}\right)$ and $\mathrm{TV}\left(u_{o}\right)$ are allowed to be large, and conversely. Then, this result may be compared with the well-known result of global existence of weak solutions for the $p$-system [38].

The proof in [3] exploits the wave-front tracking algorithm [9,19]. If the total variation of the initial data is small, then the global existence of solutions is a consequence of well-known results. We refer to [7] for a proof different from that given in [3]; in particular, in [7] the front-tracking algorithm is applied jointly to a 
path-decomposition method. The paper [39] contains an interesting analysis of the wave-pattern interactions for (4) but the proof of the global existence of solutions by the Glimm scheme seems incomplete. In the case $\pi(v, \gamma)=v^{-\gamma}$, with $\gamma>1$ replacing $\lambda$, the global existence of weak solutions for initial data with suitably bounded total variation have been proved in $[25,26]$, both by the Glimm and the front-tracking scheme. We also quote [8] for a global existence result of solutions, by compensated compactness, to the analogue of (1) in Eulerian coordinates.

Based on the analysis of system (4), the complete model (1) is then considered, where the source term is added by time discretization. We focus on the regimes $\lambda \sim 0$ and $\lambda \sim 1$.

We shall quote and describe a result [4] stating that global solutions for (1), (5) exist for a large class of initial data having suitably bounded total variation. A simple calculation shows that the system (1) is neither diagonally dominant [20] nor it satisfies the Shizuta-Kawashima condition in the hyperbolic framework [23]. As a consequence, no previously known result directly applies to the system (1).

Once the global existence of solutions to the Cauchy problem (1), (5) has been proved, we shall investigate the relaxation limit $\tau \rightarrow 0$. We shall show that for initial data close to the stable pure-phase regions, namely, either $\lambda=0$ and $p>p_{e}$ or $\lambda=1$ and $p<p_{e}$, the solutions of (1)-(5) converge to solutions of the $p$-systems

$$
\left\{\begin{array} { l l } 
{ v _ { t } - u _ { x } } & { = 0 , } \\
{ u _ { t } + p ( v , 0 ) _ { x } = 0 , }
\end{array} \quad \left\{\begin{array}{ll}
v_{t}-u_{x} & =0 \\
u_{t}+p(v, 1)_{x} & =0
\end{array}\right.\right.
$$

respectively.

We notice that the source term of (1) vanishes not only at $\lambda=0$ and $\lambda=1$ (where (1) reduces to (7)) but also at $p=p_{e}$. In that case by (1) we obtain

$$
p=p_{e}, \quad u=\text { const. }, \quad \lambda=\lambda(x)
$$

The analysis of the relaxation limit for initial data close to the equilibrium pressure is under investigation. For this case, a different approach to the treatment of the source term could be more suitable: a well-balanced approach (WE), that here consists of localizing the source term in space rather that in time, as done instead with the fractional step (FS) method. In the case of $\lambda \sim 0$, the FS approach is more convenient because it is possible to prove the exponential decay of the source term. For $p \sim p_{e}$, the WE method could be better effective since such a very strong decay does not appear to be available.

A comparison of error estimates for the two methods, in the case of a scalar equation with source term, has been recently studied in [5].

These results provide then a rigorous, even if partial, justification of the analysis that was done in [15]. In that paper, the Riemann problem for the two first equations in $(1)$ under the constraint $\left(p-p_{e}\right) \lambda(\lambda-1)=0$ was considered; such a model is formally deduced by (1) by setting $\tau=0$.

The paper is organized as follows. In Section 1 we discuss in detail several stability conditions concerning the homogeneous system (4), namely, Majda stability condition, the finiteness, BV and $\mathbf{L}^{1}$ conditions [30,34, 41]. Each of them is stricter than the previous one. We show that each shock or contact discontinuity is Majda stable and, indeed, that the finiteness condition hold. However, the BV stability condition is not satisfied by every Riemann solution, analogously to what happens to the system of nonisentropic gasdynamics, for $\gamma$ close to 1 , and in contrast to the $p$-system.

In Section 2 we introduce the main results proved in [4] about the existence of globally defined solutions to (1) and the relaxation limit $\tau \rightarrow 0$. The latter results show that in a vicinity of the stable branches of the pressure, associated to the pure phases $\lambda=0$ and $\lambda=1$, the reaction term drives the system toward a reduced $2 \times 2$ system, where the third equation is missing and the pressure is either $p(v, 0)$ or $p(v, 1)$, respectively. We emphasize that these results concern relaxation limits of weak solutions.

In Section 2 we also show that the subcharacteristic condition [36] holds, in spite of the fact that we make no explicit use of it in the proofs. Moreover, we discuss the Shizuta-Kawashima condition in the hyperbolic setting [23] for system (1). This condition is proved to imply the existence of smooth global solutions in presence 
of small initial data. We prove that the Shizuta-Kawashima condition precisely fails on $\lambda=0$ and $\lambda=1$, the case we considered previously.

\section{LARGE DATA ANALYSIS FOR THE HOMOGENEOUS SYSTEM}

In this section we deal with the stability of Riemann problems for the system (4) for large data; we will discuss the implications from the results in $[10,17,18,30,41]$. The results below hold for a general pressure law satisfying (2) and (3).

As we mentioned in the Introduction, the global existence of solutions to the Cauchy problem (4),(5) is well known if the total variation of the initial data (5) is sufficiently small $[9,19]$. Consider instead a Riemann datum

$$
(\bar{v}(x), \bar{u}(x), \bar{\lambda}(x))= \begin{cases}\left(v_{l}, u_{l}, \lambda_{l}\right) & \text { if } x<0 \\ \left(v_{r}, u_{r}, \lambda_{r}\right) & \text { if } x>0\end{cases}
$$

which gives rise to a solution of (4) composed by a juxtaposition of shocks, rarefactions and contact discontinuities. The jump at $x=0$ of $(\bar{v}(x), \bar{u}(x), \bar{\lambda}(x))$ is not required to be small.

The term stability is understood, roughly speaking, in the following sense (we refer to the papers mentioned above for a more precise discussion). Consider a datum $(\tilde{v}(x), \tilde{u}(x), \tilde{\lambda}(x))$ having small total variation. The issue is whether system (4) still has a global solution when the perturbed initial datum

$$
(\bar{v}(x), \bar{u}(x), \bar{\lambda}(x))+(\tilde{v}(x), \tilde{u}(x), \tilde{\lambda}(x))
$$

is imposed.

In the case that the Riemann problem generated by (8) consists of a single wave, then Majda's stability condition [34] is needed, see $[12,17,18]$.

More generally, assume that the Riemann problem is solved by $m$ (large) waves, with $2 \leq m \leq n$, where $n$ is the size of the system under consideration. In this case, the requirement that each single wave satisfies Majda stability condition is not a sufficient condition to obtain a self-similar solution in a neighborhood of $\mathcal{U}_{l} \times \mathcal{U}_{r}$, where $\mathcal{U}_{l}$ and $\mathcal{U}_{r}$ are neighborhoods of $\left(v_{l}, u_{l}, \lambda_{l}\right)$ and $\left(v_{r}, u_{r} \lambda_{r}\right)$, respectively. To this aim the finiteness condition $[30,31]$ is needed, which ensures that any Riemann problem in $\mathcal{U}_{l} \times \mathcal{U}_{r}$ has a self-similar solution consisting of $m$ large waves (associated to the same characteristic modes of the previous ones) and $n-m$ small waves. Moreover, the solution is unique in this class.

A more restrictive condition must be required to prove the existence of global solutions [10,30,40,41]. The aim of such a condition, called $B V$ stability condition, is to avoid the amplification of the small waves trapped into the wave pattern.

A third and still stricter condition (the $\mathbf{L}^{1}$ stability condition) is used to proved the continuous dependence on the initial data $[10,30]$. We warn the reader that the terminology is far from being unique: the finiteness condition in [41] corresponds to the BV stability condition in [30]. All of these conditions are independent of the choice of the strengths of the waves.

We now check whether Majda condition, the finiteness condition and the BV stability condition hold for system (4). The former two are proved to hold, the latter does not; as a consequence, we do not deal with the $\mathbf{L}^{1}$ stability condition. In the following, for sake of clarity, we use notation as in [41].

We write for short $U=(v, u, \lambda)$ and $f(U)=(-u, p, 0)$; moreover, we denote

$$
c=\sqrt{-p_{v}} \quad \text { and } \quad b=p_{\lambda} .
$$

The characteristic speeds for (4) under (2) and (3) are

$$
e_{-}=-c, \quad e_{0}=0, \quad e_{+}=c,
$$


with right eigenvectors

$$
R_{-}=\left(\begin{array}{c}
1 \\
c \\
0
\end{array}\right), \quad R_{0}=\left(\begin{array}{c}
b \\
0 \\
c^{2}
\end{array}\right), \quad R_{+}=\left(\begin{array}{c}
-1 \\
c \\
0
\end{array}\right) .
$$

We notice that $R_{ \pm}$are partially normalized to give $\nabla e_{ \pm} \cdot R_{ \pm}>0$. The normalized left eigenvectors are

$$
L_{-}=\frac{1}{2 c}\left(c, 1,-\frac{b}{c}\right), \quad L_{0}=\frac{1}{c^{2}}(0,0,1), \quad L_{+}=\frac{1}{2 c}\left(-c, 1, \frac{b}{c}\right) .
$$

Our first result concerns the stability of single shocks or contact discontinuities.

Lemma 1.1. Assume (2) and (3). Then, both shocks and contact discontinuities of system (4) are stable in the sense of Majda.

Proof. Denote by $U_{-}, U_{+}$the states at the left, right of the discontinuity, respectively; and by $[U]=([v],[u],[\lambda])=$ $U_{+}-U_{-}$the jump across it. With a slight abuse of notation, we write - shock wave or + shock wave for a shock associated to the eigenvalue $e_{-}$or $e_{+}$, respectively.

A - shock wave of speed $s$ is stable in the sense of Majda if $s$ is not an eigenvalue of $D f\left(U_{ \pm}\right)$and the matrix

$$
\left([U], R_{0}\left(U_{+}\right), R_{+}\left(U_{+}\right)\right)=\left(\begin{array}{ccc}
{[v]} & b_{+} & -1 \\
{[u]} & 0 & c_{+} \\
0 & c_{+}^{2} & 0
\end{array}\right)
$$

is not singular. The determinant vanishes if and only if $c_{+}[v]+[u]=0$; however, such an equality never holds because of the first Rankine-Hugoniot condition and the fact that $s$ never equals $-c_{+}$. The result for the + shock wave is completely similar.

About Majda stability condition for contact discontinuities we can proceed as in [41]. We write (4) as

$$
\begin{cases}p_{t}+c^{2} u_{x} & =0 \\ u_{t}+p_{x} & =0 \\ \lambda_{t} & =0\end{cases}
$$

Then the eigenvector $R_{0}$ becomes constant (and equal to $\left.{ }^{t}(0,0,1)\right)$ and the stability condition requires that the matrix

$$
\left(\begin{array}{ccc}
c_{-} & 0 & c_{+} \\
1 & 0 & -1 \\
0 & 1 & 0
\end{array}\right)
$$

is not singular. This clearly holds because its determinant is $c_{+}+c_{-} \neq 0$.

We now consider the BV stability condition. We recall that this condition holds for the $p$-system if the vacuum is not formed in the Riemann problem ( $[10,41])$. On the other hand, the BV stability condition is satisfied for the system of nonisentropic gasdynamics if, furthermore, $\gamma>\gamma_{0} \sim 1.015$ ( [41]).

System (4) behaves similarly to the nonisentropic gasdynamics system; BV stability condition fails in presence of very large waves.

Lemma 1.2. Assume (2) and (3). Then, the BV stability condition does not hold for system (4).

Proof. Arguing as in [41] it suffices to check the BV stability condition (called finiteness condition in [41]) in the case of a pattern formed by a - shock, a contact discontinuity, a + shock.

We use the notation in [41]. The states between two large waves are numbered from 0 to 3 as in Figure 2. Therefore, for instance, $U_{1}$ is the state between the - shock and the contact discontinuity, $a_{1}=a\left(\lambda_{1}\right)$, $c_{1}=\sqrt{-p_{v}\left(v_{1}, \lambda_{1}\right)}$. The jump of $U$ across the - shock is denoted by $[U]_{-}$and analogously for the jump across the + shock and the contact discontinuity. The speeds of the \pm shocks are denoted by $s_{ \pm}$. 


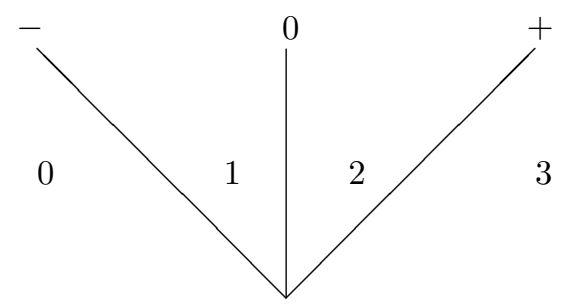

FiguRE 2. States for the Riemann problem.

We now compute the following matrices; they are related to the strengths of the waves produced by the interaction of a wave with one of the waves of the pattern.

- The reflection matrix at the - shock:

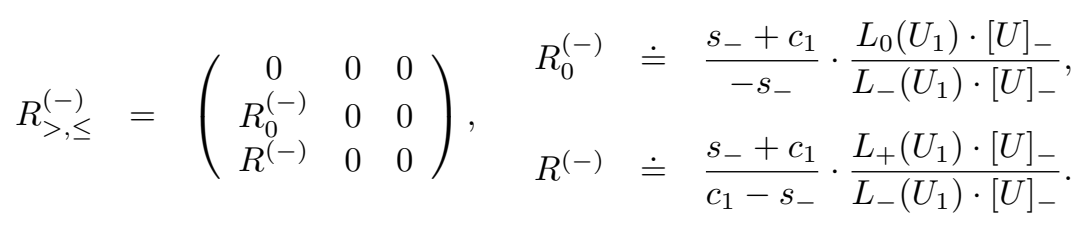

- The reflection matrix at the + shock:

$$
R_{<, \geq}^{(+)}=\left(\begin{array}{ccc}
0 & 0 & R^{(+)} \\
0 & 0 & R_{0}^{(+)} \\
0 & 0 & 0
\end{array}\right), \quad \begin{aligned}
& R^{(+)} \doteq \frac{c_{2}-s_{+}}{s_{+}+c_{2}} \cdot \frac{L_{-}\left(U_{2}\right) \cdot[U]_{+}}{L_{+}\left(U_{2}\right) \cdot[U]_{+}} \\
& R_{0}^{(+)} \doteq \frac{c_{2}-s_{+}}{s_{+}} \cdot \frac{L_{0}\left(U_{2}\right) \cdot[U]_{+}}{L_{+}\left(U_{2}\right) \cdot[U]_{+}}
\end{aligned}
$$

- The reflection matrices at the contact discontinuity:

$$
\begin{aligned}
& R_{>,<}^{(0)}=\left(\begin{array}{ccc}
0 & 0 & 0 \\
0 & 0 & 0 \\
R_{+}^{(0)} & 0 & 0
\end{array}\right), \quad R_{+}^{(0)} \doteq-\frac{L_{+}\left(U_{2}\right) \cdot R_{-}\left(U_{1}\right)}{L_{-}\left(U_{2}\right) \cdot R_{-}\left(U_{1}\right)}, \\
& R_{<,>}^{(0)}=\left(\begin{array}{ccc}
0 & 0 & R_{-}^{(0)} \\
0 & 0 & 0 \\
0 & 0 & 0
\end{array}\right), \quad R_{-}^{(0)} \doteq-\frac{L_{-}\left(U_{1}\right) \cdot R_{+}\left(U_{2}\right)}{L_{+}\left(U_{1}\right) \cdot R_{+}\left(U_{2}\right)}
\end{aligned}
$$

- The transmission matrices at the contact discontinuity:

$$
\begin{aligned}
T_{<,<}^{(0)}= & \left(\begin{array}{ccc}
T_{+}^{(0)} & 0 & 0 \\
0 & 0 & 0 \\
0 & 0 & 0
\end{array}\right), \quad T_{+}^{(0)} \doteq \frac{1}{L_{-}\left(U_{2}\right) \cdot R_{-}\left(U_{1}\right)}, \\
T_{>,>}^{(0)}= & \left(\begin{array}{ccc}
0 & 0 & 0 \\
0 & 0 & 0 \\
0 & 0 & T_{-}^{(0)}
\end{array}\right), \quad T_{-}^{(0)} \doteq \frac{1}{L_{+}\left(U_{1}\right) \cdot R_{+}\left(U_{2}\right)} .
\end{aligned}
$$

We denote by $|A|$ the matrix deduced from a matrix $A$ by taking the absolute values of the coefficients. 
The BV stability condition for the pattern formed by the - shock and the contact discontinuity states that the eigenvalues of the matrix $\left|R_{>, \leq}^{(-)} R_{<,>}^{(0)}\right|$ are less than 1 . This means that

$$
\left|R^{(-)} R_{-}^{(0)}\right|<1
$$

Next, to compute the BV stability condition for the $(-, 0)$ combination and the + shock we first need to compute the effective external reflection matrix $R_{o,>, \leq}$ of that combination. By arguing as in [41, (5.35)] the BV stability condition for $(-, 0)$ combination and the + shock turns out to be

$$
\left(\left|R_{+}^{(0)}\right|+\frac{\left|T_{-}^{(0)}\right|\left|R^{(-)}\right|\left|T_{+}^{(0)}\right|}{1-\left|R^{(-)}\right|\left|R_{-}^{(0)}\right|}\right)\left|R^{(+)}\right|<1
$$

We must check whether (10) and (11) jointly hold. For states 1 and 2 we have

$$
u_{1}=u_{2}, \quad p_{1}=p_{2}, \quad \lambda_{1}-\lambda_{2} \text { arbitrary }
$$

Then

and

$$
R_{ \pm}^{(0)}= \pm \frac{c_{2}-c_{1}}{c_{1}+c_{2}}
$$

As in [41], define

$$
T_{+}^{(0)}=\frac{2 c_{2}}{c_{1}+c_{2}}, \quad T_{-}^{(0)}=\frac{2 c_{1}}{c_{1}+c_{2}} .
$$

$$
Q=\left|R_{ \pm}^{(0)}\right|=\frac{\left|c_{2}-c_{1}\right|}{c_{2}+c_{1}} \in[0,1)
$$

Write for simplicity

Then (10) and (11) become

$$
A=\left|R^{(-)}\right|, \quad B=\left|R^{(+)}\right|
$$

$$
\begin{aligned}
A Q & <1 \\
\left(Q+\frac{\left(1-Q^{2}\right) A}{1-Q A}\right) B & <1
\end{aligned}
$$

Now we compute the values of $A, B$. About the + shock, we have

$$
s_{+}\left(v_{3}-v_{2}\right)+\left(u_{3}-u_{2}\right)=0, \quad p_{3}-p_{2}=s_{+}\left(u_{3}-u_{2}\right),
$$

and

$$
[U]_{+}=\left(v_{3}-v_{2}\right)\left(\begin{array}{c}
1 \\
-s_{+} \\
0
\end{array}\right)
$$

Therefore, recalling (9), one finds that

$$
R^{(+)}=\frac{c_{2}-s_{+}}{s_{+}+c_{2}} \cdot \frac{L_{-}\left(U_{2}\right) \cdot[U]_{+}}{L_{+}\left(U_{2}\right) \cdot[U]_{+}}=-\left(\frac{c_{2}-s_{+}}{s_{+}+c_{2}}\right)^{2}
$$

Since one has

$$
s_{+}=\sqrt{-\frac{p_{3}-p_{2}}{v_{3}-v_{2}}}, \quad c_{2}=\sqrt{-p_{v}\left(U_{2}\right)}
$$


with $v_{3}>v_{2}$ and $c_{2}>s_{+}$(by Lax conditions), we conclude that $B<1$ and that can take values arbitrarily close to 1 , for $v_{3}$ large.

Similarly, one can compute that

$$
R^{(-)}=\frac{c_{1}+s_{-}}{c_{1}-s_{-}} \cdot \frac{L_{+}\left(U_{1}\right) \cdot[U]_{-}}{L_{-}\left(U_{1}\right) \cdot[U]_{-}}=-\left(\frac{c_{1}+s_{-}}{c_{1}-s_{-}}\right)^{2}
$$

with $s_{-}=-\sqrt{-\left(p_{1}-p_{0}\right) /\left(v_{1}-v_{0}\right)}>-c_{1}$.

As a consequence, $A$ and $B$ can take any value in $(0,1)$. Then (13) is certainly satisfied, while requiring that (14) is valid for all $B \in(0,1)$ is equivalent to

$$
Q+\frac{\left(1-Q^{2}\right) A}{1-Q A}<1
$$

which in turn is equivalent to $A(1+2 Q)<1$, that is not satisfied by all Riemann patterns.

Remark 1.3. The term $Q$, defined at (12), has an important meaning in the case (6), that is for $p=A(\lambda) / v$. Indeed, set $a(\lambda)=\sqrt{A(\lambda)}$ and $a_{1,2}=a\left(\lambda_{1,2}\right)$. By using (6), one can find that

$$
Q=\frac{\left|a_{2}-a_{1}\right|}{a_{2}+a_{1}}=\frac{1}{2} \epsilon_{0}
$$

(in particular, it does not depend on $v$ ), where $\epsilon_{0}$ is the strength of the contact discontinuity used in [2,3]. This choice was done in order to reach optimal bounds on the interaction estimates between contact discontinuities and genuinely nonlinear waves.

We now briefly comment on Lemma 1.2. The failure of the BV stability condition means that there are some Riemann problems, giving rise to three waves, that do not satisfy the BV stability conditions (13)-(14). Of course, the above calculations give information about the Riemann problems that do satisfy the BV stability conditions.

We warn the reader that the failure of the BV stability condition does not necessarily mean that waves with infinite total strength are produced because of the interactions, because the effect of cancelations is ignored by that condition. For a precise discussion of this issue we refer the reader to [41, Remark 3, page 331].

We notice that condition (13) always holds. This means that the configuration shock-contact discontinuity (two large waves) always satisfies the BV stability condition. Then, the failure of the BV stability condition in the three-waves configuration formed by shock-contact discontinuity-shock ensues from the amplification of the waves trapped within the cone formed by the two shocks.

At last, we investigate whether the finiteness condition stated in [30] holds.

Lemma 1.4. Assume (2) and (3). Then, the finiteness condition holds for system (4).

Proof. Using the notation introduced in the proof of Lemma 1.2, we introduce the matrix [30, (32)]

$$
\left(\begin{array}{cccccc}
0 & 0 & a & 0 & 0 & 0 \\
0 & 0 & b & 0 & 0 & 0 \\
0 & c & 0 & 0 & e & 0 \\
0 & d & 0 & 0 & f & 0 \\
0 & 0 & 0 & g & 0 & 0 \\
0 & 0 & 0 & h & 0 & 0
\end{array}\right)=\left(\begin{array}{cccccc}
0 & 0 & R_{0}^{(-)} & 0 & 0 & 0 \\
0 & 0 & R^{(-)} & 0 & 0 & 0 \\
0 & R_{-}^{(0)} & 0 & 0 & T_{+}^{(0)} & 0 \\
0 & T_{-}^{(0)} & 0 & 0 & R_{+}^{(0)} & 0 \\
0 & 0 & 0 & R^{(+)} & 0 & 0 \\
0 & 0 & 0 & R_{0}^{(+)} & 0 & 0
\end{array}\right) .
$$

The finiteness condition requires that 1 is not an eigenvalue of the above matrix, i.e., that

$$
(1-b c)(1-f g) \neq b e d g .
$$


Hence [30, (81)]

$$
\left(R^{(-)}-R^{(+)}\right) \frac{c_{1}-c_{2}}{c_{1}+c_{2}}+R^{(-)} R^{(+)} \neq 1 .
$$

The above condition is always satisfied. Indeed, the function

$$
f(A, B ; Q)=(B-A) Q+A B-1
$$

never vanishes for $(A, B, Q)$ such that

$$
\begin{gathered}
A=-R^{(-)} \in[0,1), \quad B=-R^{(+)} \in[0,1), \\
Q=\frac{c_{1}-c_{2}}{c_{1}+c_{2}} \in(-1,1) .
\end{gathered}
$$

Thus (15) holds.

\section{MAIN RESULTS}

In this section we assume that (6) holds, so that $p(v, \lambda)=A(\lambda) / v$. We focus on the case where $\lambda \sim 0$ and $p$ is larger then $p_{e}$, namely, when data are close to the stable liquid branch. The case when $\lambda=1$ and $p$ is less than $p_{e}$, corresponding to the stable vapor branch, is dealt analogously. We refer to [4] for the proofs of the statements in this section.

\subsection{Global existence for $\tau>0$ fixed}

From the equation

$$
\lambda_{t}=\frac{1}{\tau}\left(p-p_{e}\right) \lambda(\lambda-1),
$$

the sign of $\left(p-p_{e}\right)$ determines the behavior of the equation for $\lambda$. Indeed, provided that

$$
p-p_{e} \geq c>0, \quad \lambda \leq \mu<1
$$

then we get

$$
\lambda_{t} \leq-\frac{c}{\tau}(1-\mu) \lambda
$$

and the contribution of the source term decays exponentially in time.

Theorem 2.1. For $\tau>0$ fixed, assume that

$$
0<\underline{c} \leq p_{o}(x)-p_{e} \leq \bar{c} \quad \forall x
$$

for some constants $0<\underline{c}<\bar{c}$, that $p_{o} \in B V(\mathbb{R})$ and that

$$
\text { Tot.Var. }\left(\log p_{o}\right)+\frac{1}{a(0)} \text { Tot.Var. } u_{o}<\log p_{o}(-\infty)-\log p_{e} .
$$

Then if

$$
\left\|\lambda_{o}\right\|_{\infty} \leq \mu \quad \text { and } \quad \text { Tot.Var. } \lambda_{o} \leq \mu
$$

with $\mu$ sufficiently small, the Cauchy problem (1), (5) has a weak entropy solution $(v, u, \lambda)$ defined for $t \geq 0$. Furthermore, for suitable constants $0<\underline{c}_{1}<\bar{c}_{1}$ and $c_{2}>0$, one has for every $x \in \mathbb{R}, t>0$

$$
\underline{c}_{1} \leq p(x, t)-p_{e} \leq \bar{c}_{1}, \quad 0 \leq \lambda(x, t) \leq\left\|\lambda_{o}\right\|_{\infty} \mathrm{e}^{-c_{2} \frac{t}{\tau}}
$$

and the bounds on Tot.Var. $(v, u, \lambda)(\cdot, t)$ are independent of $\tau$. 
The proof of Theorem 2.1 relies on a fractional step technique for the treatment of the source term in (1). It is combined with a wave-front tracking algorithm for the approximation of solutions to (4).

The proof begins with a suitable approximation of the initial data; then let $\Delta t>0$ be the mesh size in time and set $t_{n}=n \Delta t$. The solution is constructed by recursion. In each time interval $\left[t_{n}, t_{n+1}\right)$ the solution is determined by solving suitable Riemann problems of the homogeneous system (4); then, at times $t_{n}$ the solution is updated as follows, see Figure 3:

$$
\begin{aligned}
(v, u)\left(\cdot, t_{n}\right) & =(v, u)\left(\cdot, t_{n}-\right) \\
\lambda\left(\cdot, t_{n}\right) & =\left.\lambda(\cdot, t)\left\{1-\frac{\Delta t}{\tau}\left(p(\cdot, t)-p_{e}\right)(1-\lambda(\cdot, t))\right\}\right|_{t=t_{n}-} .
\end{aligned}
$$

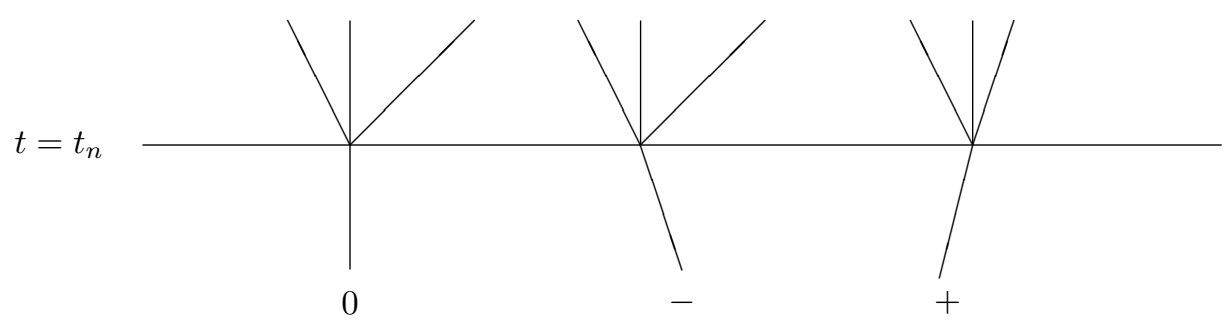

FiguRE 3. The fractional step scheme; the labels $-, 0,+$ denote the wave family.

The main point in the proof consists in showing that a suitable functional $\mathcal{F}$ is decreasing. The functional $\mathcal{F}$ essentially consists of two parts. One part controls the variation in the conservative steps and is defined as

$$
F(t)=L_{\xi}+K Q
$$

where

$$
\begin{aligned}
L_{\xi}(t) & =\sum_{\gamma_{ \pm}>0}\left|\gamma_{ \pm}\right|+\xi \sum_{\gamma_{ \pm}<0}\left|\gamma_{ \pm}\right|+K_{n p} \sum_{\gamma \in \mathcal{N} \mathcal{P}}|\gamma| \\
Q(t) & =\sum_{\left(\gamma_{ \pm}, \delta_{0}\right) \in \mathcal{A}}\left|\gamma_{ \pm} \delta_{0}\right| .
\end{aligned}
$$

Here, $\xi, K$ and $K_{n p}$ are positive parameters to be determined. The summations in (17) are performed over all shock $(+)$ or rarefaction $(-)$ waves in the solution at time $t$; remark the weight $\xi$ for shock waves that was inspired by [6]. The notation $\mathcal{N} \mathcal{P}$ indicates the nonphysical waves, that are introduced for having the scheme well-defined.

In (18) the notation $\left(\gamma_{ \pm}, \delta_{0}\right) \in \mathcal{A}$ means that the sum is performed over all \pm waves $\gamma_{ \pm}$that are approaching a phase wave $\delta_{0}$, i.e., the waves $\gamma_{+}\left(\gamma_{-}\right)$at the left (resp., right) of $\delta_{0}$.

The functional $F$ was proved in [3] to be decreasing in time. Here, however, a control at the time steps is needed and, then, some terms need to be added to $F$ to balance its possible increase at such times. The final functional is

$$
\mathcal{F}=F+K_{1} \Lambda+K_{2} T+K_{3} Q_{2}
$$

where

$$
\Lambda(t)=\|\lambda(\cdot, t)\|_{\infty}, \quad T(t)=\operatorname{Tot} \cdot \operatorname{Var} . \lambda(\cdot, t), \quad Q_{2}(t)=\sum_{x_{i}<x_{j}}\left|\delta_{0}^{i} \delta_{0}^{j}\right|
$$


In the sum in $(20), \delta_{0}^{i}$ denotes the strength of the 0 -wave located at point $x_{i}$ at time $t$. Here, again, the constants $K_{1}, K_{2}, K_{3}$ have to be determined.

Lemma 2.2. For a suitable choice of the above parameters, the functional $\mathcal{F}$ is decreasing in time.

\subsection{The limit $\tau \rightarrow 0$}

Here we consider the relaxation limit, $\tau \rightarrow 0$. For small values of $\lambda$, the solutions obtained in Theorem 2.1 are proved to converge to solutions of the limit system $(7)_{1}$.

Theorem 2.3. For $\tau>0$, let the initial data

$$
(v, u, \lambda)(x, 0)=\left(v_{o}^{\tau}(x), u_{o}^{\tau}(x), \lambda_{o}^{\tau}(x)\right)
$$

satisfy the bounds of Theorem 2.1 uniformly in $\tau$. Assume that

$$
v_{o}^{\tau} \rightarrow v_{o}, \quad u_{o}^{\tau} \rightarrow u_{o} \quad \text { in } \quad L_{l o c}^{1}(\mathbb{R}), \quad \text { as } \tau \rightarrow 0
$$

Let $\left(v^{\tau}, u^{\tau}, \lambda^{\tau}\right)(x, t)$ be the weak entropic solution provided by Theorem 2.1.

Then there exists a (sub) sequence $\tau_{n} \rightarrow 0 \mathrm{~s}$. $t$.

$$
\begin{aligned}
\lambda^{\tau_{n}} & \rightarrow 0 & \text { in } L_{\text {loc }}^{1}(\mathbb{R} \times(0, \infty)) \\
\left(v^{\tau_{n}}, u^{\tau_{n}}\right) & \rightarrow(\widetilde{v}, \widetilde{u}) & \text { in } L_{\text {loc }}^{1}(\mathbb{R} \times[0, \infty)),
\end{aligned}
$$

where $(\widetilde{v}, \widetilde{u})$ is a weak solution for $t \geq 0$ to the Cauchy problem

$$
\left\{\begin{array} { l l } 
{ v _ { t } - u _ { x } } & { = 0 } \\
{ u _ { t } + p ( v , 0 ) _ { x } } & { = 0 , }
\end{array} \quad \left\{\begin{array}{l}
v(x, 0)=v_{o}(x) \\
u(x, 0)=u_{o}(x) .
\end{array}\right.\right.
$$

Moreover consider any smooth, convex entropy $\eta(v, u, \lambda)$ for system (1), that satisfies

$$
\partial_{\lambda} \eta \cdot\left(p-p_{e}\right) \geq 0
$$

Let $q(v, u, \lambda)$ be the corresponding entropy flux. Then $(\widetilde{v}, \widetilde{u})$ is entropic w.r.t. the entropy pair $\widetilde{\eta}=\eta(v, u, 0)$, $\widetilde{q}=q(v, u, 0)$.

There are two key points in the proof of Theorem 2.3. First, the bounds on the total variation of $U$ provided in Theorem 2.1 are independent of $\tau$. This makes the limit $\tau \rightarrow 0$ possible for the variables $v$ and $u$. Second, the following estimate for $L^{1}$ spatial norm for the evolution of $\lambda$

$$
\|\lambda(\cdot, t)-\lambda(\cdot, s)\|_{L^{1}([a, b])} \leq L_{\tau}(t-s+\Delta t)
$$

is proved. That estimate, in turn, allows to prove the relaxation limit for $\lambda$ by a diagonal argument.

Remark 2.4. Notice that the convergence of the sequence $\lambda^{\tau_{n}}$ holds only on $\mathbb{R} \times(0, \infty)$, that is, not for $t \geq 0$ in general; indeed, $\lambda_{o}^{\tau}$ is not assumed to converge as $\tau \rightarrow 0$. Nevertheless, thanks to the second equation in (16), $\lambda^{\tau}$ vanishes in the limit for $t>0$.

Remark 2.5. If $\eta(v, u, \lambda)$ satisfies (21), we say that $\eta$ is dissipative with respect to the source term in (1). Indeed, for $\tau>0$ the entropy inequality reads as

$$
\partial_{t} \eta+\partial_{x} q \leq \frac{1}{\tau} \partial_{\lambda} \eta \cdot\left(p-p_{e}\right) \lambda(\lambda-1)
$$

and the last term is $\leq 0$ if the condition (21) holds. 
Among the limit entropies $\widetilde{\eta}$, it is easy to check that we can recover the standard entropy for the $2 \times 2$ system with $\lambda=0$, that is

$$
\widetilde{\eta}(v, u)=\frac{u^{2}}{2}-A(0) \log v
$$

with corresponding entropy flux $\widetilde{q}(v, u)=\frac{A(0) u}{v}$.

Indeed, one can check that

$$
\eta(v, u, \lambda)=\frac{u^{2}}{2}-A(\lambda) \log v+\phi(\lambda), \quad q(v, u, \lambda)=\frac{A(\lambda) u}{v}
$$

is an entropy-entropy flux pair for the $3 \times 3$ system. For a suitable choice of $\phi(\lambda), \eta$ turns out to be strictly convex and dissipative in the sense given above, $(21)$. Hence, for $\tau_{n} \rightarrow 0$, the limit $(\widetilde{v}, \widetilde{u})$ is entropic with respect to the standard entropy-entropy flux $\widetilde{\eta}, \widetilde{q}$.

Remark 2.6. We discuss the validity of the subcharacteristic condition, introduced in [11] as a stability condition in the context of relaxation systems. Using the notation in (2), we recall that the characteristic speeds for system (4) are $e_{-}=-c, e_{0}=0, e_{+}=c$ with $c=a(\lambda) \sqrt{-\pi_{v}}, a(\lambda)=\sqrt{A(\lambda)}$.

Denoting by $\widetilde{e}_{ \pm}$the eigenvalues of the limit system, the subcharacteristic condition amounts in requiring that, on the equilibrium manifold, one has the following interlaced inequalities for the eigenvalues:

$$
e_{-} \leq \widetilde{e}_{-} \leq 0 \leq \widetilde{e}_{+} \leq e_{+}
$$

In our case, $e_{-}=\widetilde{e}_{-}<0<\widetilde{e}_{+}=e_{+}$, hence the condition holds.

\subsection{Sufficient conditions for global existence}

We now briefly discuss two conditions for hyperbolic systems of balance laws that lead to solutions defined globally in time.

We define

$$
g(U)=\left(0,0, \frac{1}{\tau}\left(p-p_{e}\right) \lambda(\lambda-1)\right)
$$

so that system (1) can be written as $U_{t}+f(U)_{x}=g(U)$.

First, system (1) does not satisfy the diagonal dominance condition $[6,20]$. Indeed, the the matrix $D g$ in not invertible, which is a necessary condition for diagonally dominance. Then, the global existence of weak solutions for small $B V$ initial data cannot be deduced by [20].

Second, we recall the Shizuta-Kawashima [27,42] condition in the hyperbolic framework [23]. This condition is proven in [23] to lead to the global existence of smooth solutions for small initial data. The Shizuta-Kawashima condition holds if at any equilibrium point $U_{o}$

$$
\text { ker } D g\left(U_{o}\right) \cap\left\{\text { eigenspaces of } D f\left(U_{o}\right)\right\}=\{0\} \text {. }
$$

Using the notation of Section 1 we have the following:

$$
\begin{aligned}
(D g) R_{ \pm} & =\frac{1}{\tau}\left(0,0, \mp p_{v} \lambda(\lambda-1)\right) \\
(D g) R_{0} & =\frac{1}{\tau}\left(0,0, p_{\lambda} p_{v} \lambda(\lambda-1)-p_{v}\left(p-p_{e}\right)(2 \lambda-1)\right) .
\end{aligned}
$$

Then

(i) if $\lambda=0$ or $\lambda=1$, then $R_{ \pm} \in \operatorname{ker} D g$;

(ii) if $p(v, \lambda)=p_{e}$, then $R_{ \pm}, R_{0} \notin \operatorname{ker} D g$. 
and therefore (22) is not satisfied at $\lambda=0$ or $\lambda=1$, while it is satisfied at $p=p_{e}$.

As a consequence, the result of [23] can be applied for perturbations of equilibrium data with $p=p_{e}$ and gives the global existence of smooth solutions provided that the perturbation is suitably small in the $C^{1}$ norm. An analogous result for small BV perturbations of equilibrium data is not available.

\section{REFERENCES}

[1] R. Abgrall. Generalisation of the Roe scheme for the computation of mixture of perfect gases. J. Comp. Physics, 125(12):150$160,1996$.

[2] D. Amadori and A. Corli. A hyperbolic model of multiphase flow. In Hyperbolic problems: theory, numerics, applications, pages 407-414. Springer, Berlin, 2008.

[3] D. Amadori and A. Corli. On a model of multiphase flow. SIAM J. Math. Anal., 40(1):134-166, 2008.

[4] D. Amadori and A. Corli. Global existence of BV solutions and relaxation limit for a model of multiphase reactive flow. Nonlinear Anal., 72(5):2527-2541, 2010.

[5] D. Amadori and L. Gosse. Transient $L^{1}$ error estimates for well-balanced schemes on non-resonant scalar balance laws. Technical report, University of L'Aquila, 2012.

[6] D. Amadori and G. Guerra. Global weak solutions for systems of balance laws. Appl. Math. Lett., 12(6):123-127, 1999.

[7] F. Asakura and A. Corli. Global existence of solutions by path decomposition for a model of multiphase flow. Quart. Appl. Math., to appear, 2012.

[8] F. Bereux, E. Bonnetier, and P. G. LeFloch. Gas dynamics system: two special cases. SIAM J. Math. Anal., 28(3):499-515, 1997.

[9] A. Bressan. Hyperbolic systems of conservation laws, volume 20 of Oxford Lecture Series in Mathematics and its Applications. Oxford University Press, Oxford, 2000. The one-dimensional Cauchy problem.

[10] A. Bressan and R. M. Colombo. Unique solutions of $2 \times 2$ conservation laws with large data. Indiana Univ. Math. J., 44(3):677$725,1995$.

[11] G. Q. Chen, C. D. Levermore, and T.-P. Liu. Hyperbolic conservation laws with stiff relaxation terms and entropy. Comm. Pure Appl. Math., 47(6):787-830, 1994.

[12] I.-L. Chern. Stability theorem and truncation error analysis for the Glimm scheme and for a front tracking method for flows with strong discontinuities. Comm. Pure Appl. Math., 42(6):815-844, 1989.

[13] J.-P. Cocchi, R. Saurel, and J. C. Loraud. A riemann problem based method for the resolution of compressible multimaterial flows. J. Comp. Physics, 137(2):265-298, 1997.

[14] R. M. Colombo and A. Corli. Continuous dependence in conservation laws with phase transitions. SIAM J. Math. Anal., 31(1):34-62 (electronic), 1999.

[15] A. Corli and H. Fan. The Riemann problem for reversible reactive flows with metastability. SIAM J. Appl. Math., 65(2):426-457 (electronic), 2004/05.

[16] A. Corli and H. Fan. Traveling waves of phase transitions in porous media. Appl. Anal., to appear, 2012.

[17] A. Corli and M. Sablé-Tougeron. Perturbations of bounded variation of a strong shock wave. J. Differential Equations, 138(2):195-228, 1997.

[18] A. Corli and M. Sablé-Tougeron. Stability of contact discontinuities under perturbations of bounded variation. Rend. Sem. Mat. Univ. Padova, 97:35-60, 1997.

[19] C. M. Dafermos. Hyperbolic conservation laws in continuum physics, volume 325 of Grundlehren der Mathematischen Wissenschaften [Fundamental Principles of Mathematical Sciences]. Springer-Verlag, Berlin, third edition, 2010.

[20] C. M. Dafermos and L. Hsiao. Hyperbolic systems and balance laws with inhomogeneity and dissipation. Indiana Univ. Math. J., 31(4):471-491, 1982.

[21] H. Fan. On a model of the dynamics of liquid/vapor phase transitions. SIAM J. Appl. Math., 60(4):1270-1301 (electronic), 2000.

[22] L. Gosse. Existence of $L^{\infty}$ entropy solutions for a reacting Euler system. Port. Math. (N.S.), 58(4):473-484, 2001.

[23] B. Hanouzet and R. Natalini. Global existence of smooth solutions for partially dissipative hyperbolic systems with a convex entropy. Arch. Ration. Mech. Anal., 169(2):89-117, 2003.

[24] P. Helluy and N. Seguin. Relaxation models of phase transition flows. M2AN Math. Model. Numer. Anal., 40(2):331-352, 2006.

[25] H. Holden, N. H. Risebro, and H. Sande. The solution of the Cauchy problem with large data for a model of a mixture of gases. J. Hyperbolic Differ. Equ., 6(1):25-106, 2009.

[26] H. Holden, N. H. Risebro, and H. Sande. Front tracking for a model of immiscible gas flow with large data. BIT, 50(2):331-376, 2010.

[27] S. Kawashima. Large-time behavior of solutions to hyperbolic-parabolic systems of conservation laws and applications. Proc. Roy. Soc. Edinburgh Sect. A, 106(1-2):169-194, 1987. 
[28] P. G. LeFloch. Hyperbolic systems of conservation laws. Lectures in Mathematics ETH Zürich. Birkhäuser Verlag, Basel, 2002. The theory of classical and nonclassical shock waves.

[29] R. LeVeque, D. Yee, R. P., and B. van Leer. Model systems for reacting flows. NASA-Ames University Consortium, NCA2-185, 1988.

[30] M. Lewicka. Well-posedness for hyperbolic systems of conservation laws with large BV data. Arch. Ration. Mech. Anal., 173(3):415-445, 2004.

[31] M. Lewicka. Stability conditions for strong rarefaction waves. SIAM J. Math. Anal., 36(4):1346-1369 (electronic), 2005.

[32] Y.-g. Lu and C. Klingenberg. The Cauchy problem for hyperbolic conservation laws with three equations. J. Math. Anal. Appl., 202(1):206-216, 1996.

[33] Y.-g. Lu and C. Klingenberg. The relaxation limit for systems of Broadwell type. Differential Integral Equations, 14(1):117-127, 2001.

[34] A. Majda. Compressible fluid flow and systems of conservation laws in several space variables, volume 53 of Applied Mathematical Sciences. Springer-Verlag, New York, 1984.

[35] S. Müller, P. Helluy, and J. Ballmann. Numerical simulation of a single bubble by compressible two-phase fluids. Internat. J. Numer. Methods Fluids, 62(6):591-631, 2010.

[36] R. Natalini. Recent results on hyperbolic relaxation problems. In Analysis of systems of conservation laws (Aachen, 1997), pages 128-198. Chapman \& Hall/CRC, Boca Raton, FL, 1999.

[37] T. Nishida. Global solution for an initial boundary value problem of a quasilinear hyperbolic system. Proc. Japan Acad., 44:642-646, 1968.

[38] T. Nishida and J. A. Smoller. Solutions in the large for some nonlinear hyperbolic conservation laws. Comm. Pure Appl. Math., 26:183-200, 1973.

[39] Y.-J. Peng. Solutions faibles globales pour un modèle d'écoulements diphasiques. Ann. Scuola Norm. Sup. Pisa Cl. Sci. (4), 21(4):523-540, 1994.

[40] M. Sablé-Tougeron. Stabilité de la structure d'une solution de Riemann à deux grands chocs. Ann. Univ. Ferrara Sez. VII (N.S.), 44:129-172 (1999), 1998.

[41] S. Schochet. Sufficient conditions for local existence via Glimm's scheme for large BV data. J. Differential Equations, 89(2):317354, 1991.

[42] Y. Shizuta and S. Kawashima. Systems of equations of hyperbolic-parabolic type with applications to the discrete Boltzmann equation. Hokkaido Math. J., 14(2):249-275, 1985.

[43] A. Sommerfeld. Thermodynamics and statistical mechanics. (Lectures on theoretical physics, vol. V.). Academic Press Inc., New York, 1956. Edited by F. Bopp and J. Meixner, Translated by J. Kestin.

[44] P. A. Thompson, H. Chaves, G. E. A. Meyer, Y.-G. Kim, and H.-D. Speckmann. Wave splitting in a fluid of large heat capacity. J. Fluid Mech., 185:385-414, 1987. 